\title{
Usage des graines a vocation médicinale commercialisées sur le marché de Adjarra- Kpetou dans la commune de Adjarra au sud-est du Benin
}

\author{
Rachad Kolawolé Foumilayo Mandus ALI \\ Enseignant-Chercheur, Béninois, Université d'Abomey-Calavi, Faculté des Sciences Humaines et Sociales, \\ Département de Géographie et Aménagement du Territoire, Bénin, Laboratoire de Biogéographie et Expertise \\ Environnemental. E-mail :ali.rachad@yahoo.fr; alvitomevognon@gmail.com.
}

Original submitted in on 14th February 2019. Published online at www.m.elewa.org/journals/ on 31st May 2019 https://dx.doi.org/10.4314/jab.v137i1.5

\begin{abstract}
RESUME
Objectif: Cette étude se propose de faire la synthèse des connaissances de l'usage des graines à vocation médicinale vendues sur le marché de Adjarra-kpétou au sud-est du Bénin.

Méthodologie et résultats : Les données ethnobotaniques ont été collectées auprès de 47 herboristes enquêtées de manière exhaustive dont 04 guérisseurs traditionnels aléatoirement choisis. Ces données ont été analysées à partir de l'estimation de la fréquence relative de citation $(F R C)$ et la fréquence d'implication des graines dans la constitution des recettes (CPR). Au total, 20 graines à vocation médicinales réparties en 16 genres et 11 familles ont été recensées. Ces graines sont utilisées dans la formulation de 79 recettes pour traiter 42 différentes maladies. La macération est la forme pharmaceutique la plus pratiquée (32\%). Conclusions et applications des résultats : Ces résultats ainsi obtenus permettront à l'Etat en l'occurrence, le ministre de la santé publique, de développer des actions de conservation et de vulgarisation des graines à vocation médicinale dans les marchés à travers la prise des arrêtées en vue de faire la promotion des connaissances endogènes.
\end{abstract}

Mots clés : Marché Adjarra-kpétou, ethnobotanique, herboristes, graines médicinales

Use of seeds used for medicinal purposes sold on Adjarra-kpetou market in Adjarra municipality in southeastern Benin

\begin{abstract}
Objective: This study aims to synthesize the knowledge of the use of medicinal seeds sold in the market of Adjarra-kpétou in southeastern Benin.

Methodology and results: Ethnobotanical data were collected from 47 herbalists surveyed exhaustively including 04 traditional healers randomly selected. These data were analyzed from the estimation of the relative frequency of citation (RFC) and the frequency of seed involvement in the constitution of the recipes (CPR). A total of 20 medicinal seeds divided into 16 genera and 11 families have been identified. These seeds are used in 79 recipes for the treatment of 42 different diseases. Maceration is the most popular pharmaceutical form (32\%).
\end{abstract}


Conclusions and applications of the output: These results thus obtained will enable the State, in this case, the Minister of Public Health, to develop actions of conservation and popularization of seeds for medicinal purposes in the markets through the adoption of ordersaiming at promoting endogenous knowledge.

Keywords: Adjarra-kpétou market, ethnobotany, herbalists, medicinal seeds.

\section{INTRODUCTION}

L'importance des ressources végétales naturelles n'est plus à démontrer, surtout en milieu rural où, le plus souvent, elles jouent un rôle fondamental dans la survie et le développement des populations (Gueye et al., 2012, p. 773). L'inexistence des infrastructures sanitaires, la constante augmentation des prix des produits et prestations de santé, l'inaccessibilité aux médicaments génériques constituent un problème majeur pour les populations financièrement démunies (Ngene et al., 2015, p. 8195). Du coup, l'exigence des plantes médicinales augmente de plus en plus dans le monde aussi bien dans les pays développés que sous-développés (Fatiha et Lahcen, 2015, p. 8494). C'est pourquoi Jiofack et al. $(2010$, p. 62) estiment qu'environ $80 \%$ de la population mondiale et plus de $90 \%$ de la population des pays en voie de développement font recours aux espèces végétales pour les soins de santé primaire. De plus, selon Dougnon et al. (2016, p. 17), les plantes médicinales constituent une source de soins médicaux de proximité. En conséquence, médecins et organismes de santé portent davantage un intérêt aux valeurs et à l'efficacité des traitements par les plantes, après avoir longtemps combattu la médecine traditionnelle (Mpondo et al., 2015, p. 7805). Au Bénin, une attention particulière est portée sur les graines qui représentent une partie de la plante. En effet, qu'elles soient utilisées comme nourritures, huiles cosmétiques, médicaments ou simples semences, les graines ne cessent d'accompagner quotidiennement les hommes car leur vente est non seulement une source de revenue, mais aussi un moyen de vulgarisation et de pérennisation du savoir endogène associé à l'usage des plantes médicinales. D'après. Eddouks et al. (2007, p. 195), les graines sont utilisées comme des ingrédients auxiliaires ou adjuvants, permettant de renforcer l'action thérapeutique des composantes principales des recettes et aussi de traiter les symptômes secondaires des maladies. Par ailleurs, pour documenter et ainsi pérenniser le savoir traditionnel, plusieurs travaux de recherches ethnobotaniques et/ou ethno pharmacologiques ont été entrepris un peu partout en Afrique de l'Ouest. A ce titre on peut citer, au Burkina Faso (Zerbo et al., 2007); au Bénin (Adomou et al., 2012 ; Lougbégnon et al., 2015 et. Kouchadé et al., 2016) ; au Niger (. Wezel, 2001) au Togo (Adjanohoun et al., 1986) au Nigeria (Okafor et Ham, 1999) et en Côte d'ivoire (Adjanohoun et Ake-assi, 1979 ; Koné et al., 2002 et Gnagné et al., 2017). Cependant, presque aucune étude n'a été menée de façon particulière sur l'utilisation médicinale des graines, bien que leur commerce constitue une pratique très courante aussi bien qu'au Bénin qu'ailleurs. De plus, malgré ces nombreux projets de recherches ethnobotaniques effectuées, subsistent toujours au Bénin des régions, des marchés ou encore des groupes ethniques qui n'ont jamais été l'objet de travaux sur les plantes médicinales. C'est pourquoi la présente étude porte sur l'usage des graines à vocation médicinale commercialisées dans le marché de Adjarra-Kpétou (Commune de Adjarra au Bénin). Elle s'articule autour de deux axes: dans une première phase, une enquête est entreprise auprès des herboristes pour l'inventaire et l'identification des graines à vocation médicinales vendues et dans la seconde phase, il a été recueilli toujours auprès des herboristes et des acheteurs (dont des guérisseurs traditionnels), le maximum d'informations relatives à l'utilisation thérapeutique de chacune des graines inventoriées. 


\section{MATERIEL ET METHODES}

Echantillonnage : Les enquêtes en milieu réel ont été menées selon un recensement exhaustif des herboristes du marché. Ainsi, un total de 47 étalages de graines médicinales a été visité. Ajouté à ceux-ci, 29 acheteurs dont 04 guérisseurs traditionnels ont été aléatoirement enquêtés dans le secteur d'étude. Soit un échantillonnage de 76 personnes.

Technique de collecte : Les entretiens ont été chaque fois réalisés sur la base d'un dialogue dans les langues locales Yoruba et Goun. Chaque entretien était accompagné d'une observation directe et de l'achat des graines médicinales pour identification des noms scientifiques.

Données collectées : Dans le cadre de cette étude, le profil de chaque enquêté (sexe, niveau d'instruction et ethnie) et le nom vernaculaire de chaque espèce de graine présente sur chacun des étals ont été déterminés. Les diverses maladies traitées, les recettes, les formes pharmaceutiques, les éléments d'association qui y concourent à la préparation des recettes recensées et le mode d'administration des médicaments, ainsi que la dose d'utilisation des recettes ont été déterminés à l'aide de la fiche de collecte adressé individuellement aux herboristes, acheteurs et guérisseurs traditionnels du secteur d'étude.

Identification taxonomique des graines médicinales: L'identification taxonomique des espèces de graines médicinales a été réalisée à partir de la flore analytique du Bénin (A. Akoègninou et al.,

\section{RESULTATS}

Diversité des graines médicinales commercialisées dans le marché de Adjarra-Kpétou: La présente étude a permis de recenser 20 espèces de graines médicinales vendues par les herboristes et utilisées pour traiter plusieurs maladies. Ces espèces sont reparties en 16 genres et 11 familles. Les familles les plus dominantes sont les Alliaceae (15\%), les Mimosaceae (15\%), les Sterculaceae $(15 \%)$ et des
2006) et par des experts du jardin botanique de l'Université d'Abomey-Calavi.

Méthode de traitement et d'analyse des données: Les données quantitatives collectées sont dépouillées manuellement et encodées dans une base de données conçue et traitée sous le tableur Excel 2010. Ces données ont permis de réaliser des graphiques et tableaux pour mieux illustrer les informations relatives à l'inventaire et à l'usage médicinal des graines commercialisées dans le marché de Adjarra-kpétou. Aussi, les différentes affections ont été classées en des types de maladies par un médecin de la zone d'étude.

Fréquence relative de citation: L'importance locale de chaque espèce de graine a été déterminée à partir de la fréquence relative de citation (Tardio, et PardoDe-Santayana, 2008). Elle est calculée suivant la formule :

$\mathbf{F R C}=\frac{\boldsymbol{F} \boldsymbol{c}}{\boldsymbol{N}}$ Avec: $\quad$ Fc $=$ nombre d'enquêtés ayant mentionné l'usage de l'espèce ;

$\mathrm{N}=$ nombre total d'enquêtés.

Fréquences d'implications des graines dans les recettes: La contribution de chacune des graines inventoriées, dans la constitution des recettes a été identifiée à partir de la formule de Dassou et al., (2014, p. 192) :

$\mathrm{CPR}=\frac{\mathrm{Nr}}{\mathrm{Nt}} \mathrm{x} 100$ Avec: $\mathrm{Nr}=$ nombre de recettes sollicitant l'espèce de graine. $\mathrm{Nt}=$ nombre total de recettes. Par ailleurs, cette formule a été adaptée à l'étude.

Zingiberaceae (15\%). Suivies des Hypoxydaceae (5 $\%$ ), des Antheriaceae (5\%), des Piperaceae (5\%), des Apocynaceae $(5 \%)$, des Clusiaceae $(5 \%)$ et des Myrtaceae (5\%).

Espèces inventoriées et leur fréquence de citation : Parmi les espèces inventoriées, certaines se révèlent être plus fréquemment citées. La figure 3renseigne sur la fréquence de citation de chaque graine. 


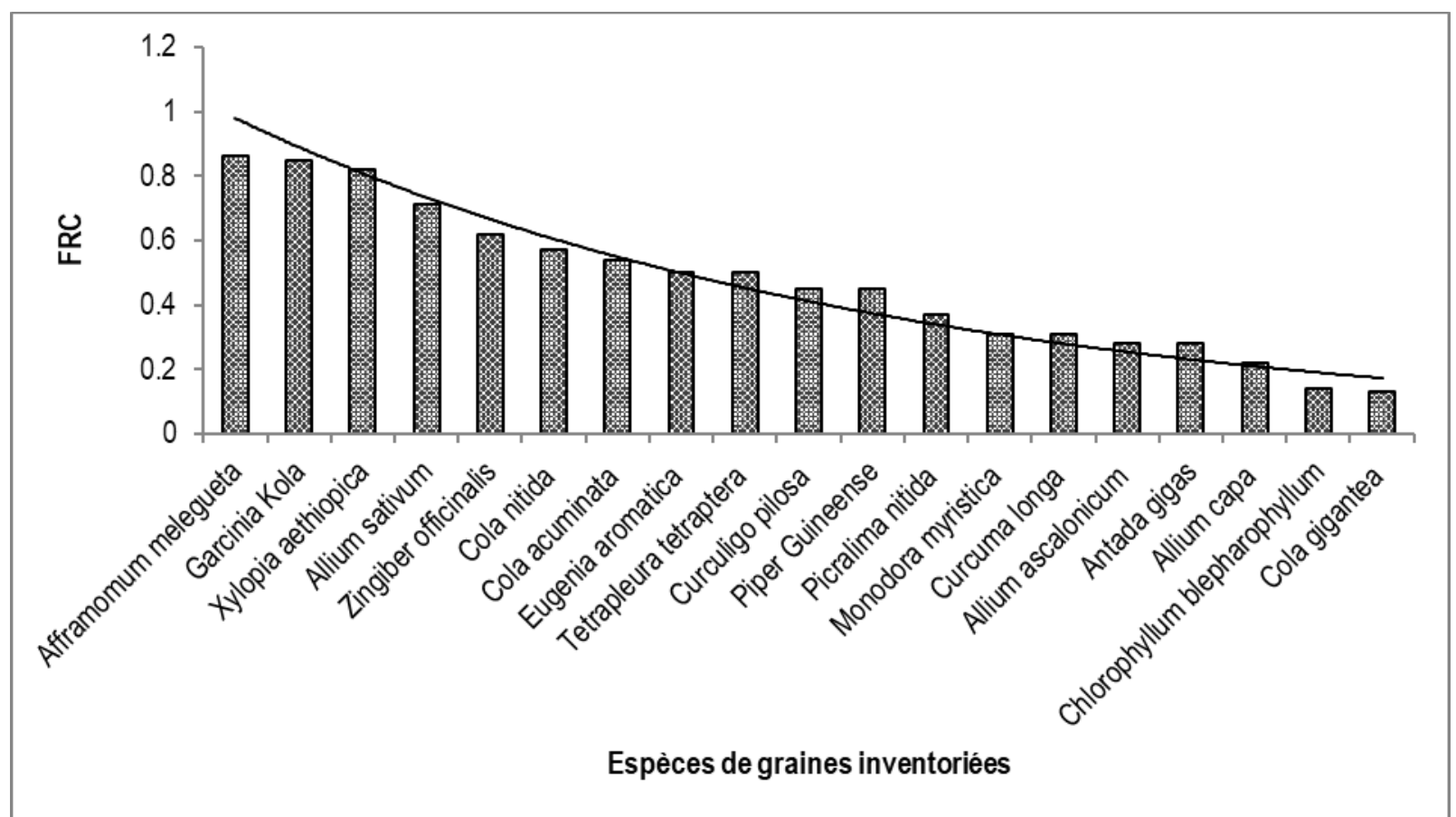

Figure 3 : Fréquence de citation des espèces de graines Source : Enquêtes de terrain, mars 2018

II ressort de l'analyse de la figure 3 que, plus de $50 \%$ des herboristes ont cité les espèces telles que: Afframomum melegueta ( $F R C=0,86)$, Garcinia kola (FRC $=0,85)$, Xylopia aethiopica (FRC $=0,82)$, Allium sativum $(F R C=0,71)$ et Zingiber officinalis $(F R C=$
0,62). Ces espèces sont fréquentes sur les étalages des herboristes. Ceci témoigne, par conséquent, leur importance thérapeutique dans le secteur d'étude. La planche 1 présente les graines les plus citées et les plus utilisées par les enquêtés.

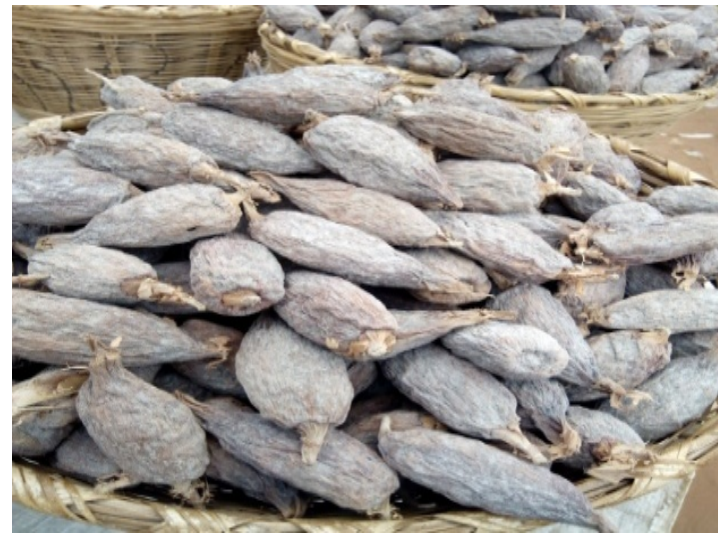

Afframomum melegueta

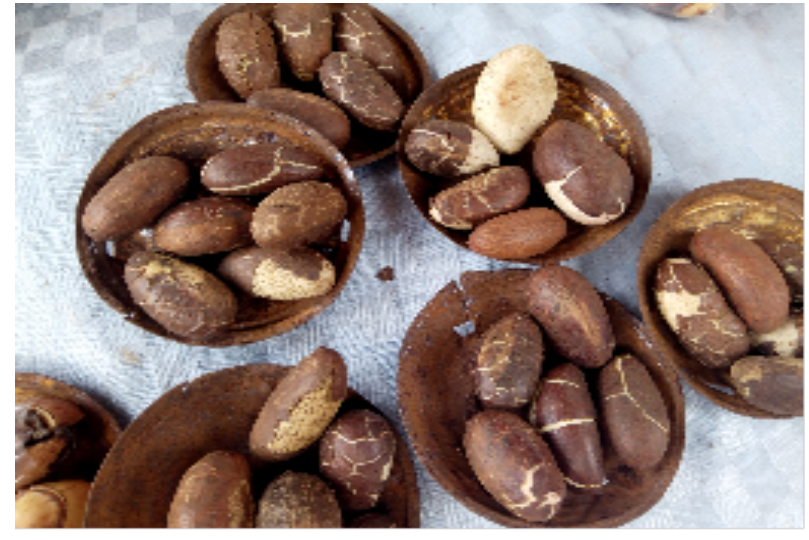

Garcinia kola 


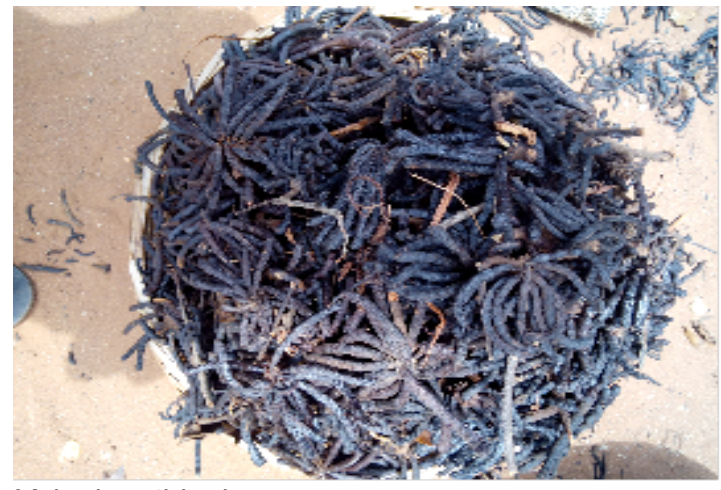

Xylopiaaethiopica

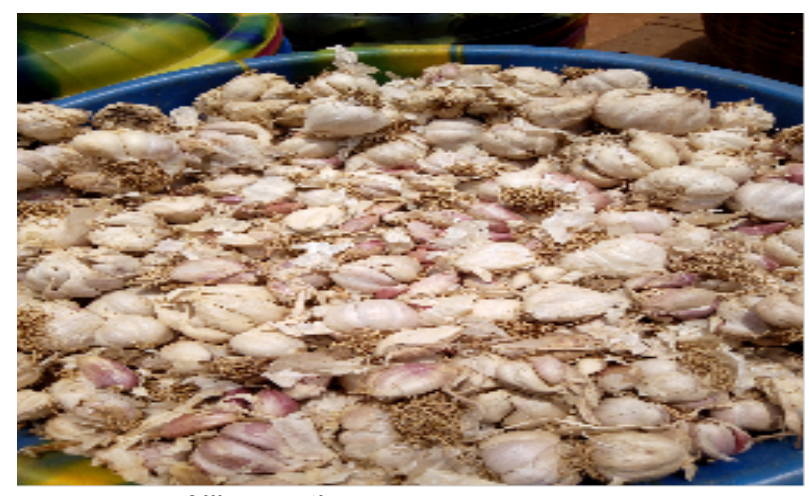

Allium sativum
Fréquence d'implication des graines dans les recettes: Au total, soixante-dix-neuf (79) différentes recettes ont été recensées au cours des séries d'enquêtes effectuées. Par ailleurs, il s'avère nécessaire de préciser que certaines espèces de graines sont plus impliquées dans la constitution de ces recettes. Le tableau 1 indique la fréquence d'implication de chacune des espèces de graines recensées sur le terrain.

Tableau 1 : Fréquence d'implication de chacune des espèces de graines (CPR) recensée dans la constitution des recettes

\begin{tabular}{|l|l|l|l|c|c|}
\hline $\mathbf{N}^{\mathbf{0}}$ & \multicolumn{1}{|c|}{$\begin{array}{c}\text { Noms vernaculaire } \\
\text { (Yorouba) }\end{array}$} & \multicolumn{1}{|c|}{ Noms scientifiques } & Nr & CPR \\
\hline $\mathbf{0 1}$ & Sielede & Acacia siberiana & Mimosaceae & 02 & 2,53 \\
\hline $\mathbf{0 2}$ & Ataré & Afframomum melegueta & Zingiberaceae & 12 & 15,18 \\
\hline $\mathbf{0 3}$ & Aloubosawèwè & Allium ascalonicum & Alliaceaea & 09 & 11,39 \\
\hline $\mathbf{0 4}$ & Aloubosaoko & Allium cepa & Alliaceaea & 07 & 8,86 \\
\hline $\mathbf{0 5}$ & Ayo & Allium sativum & Alliaceaea & 07 & 8,86 \\
\hline $\mathbf{0 6}$ & Gbagblakoun & Antada gigas & Mimosaceae & 01 & 1,26 \\
\hline $\mathbf{0 7}$ & Baani & Chlorophyllum bleralophyyllum & Antheriaceae & 02 & 2,53 \\
\hline $\mathbf{0 8}$ & Obi olujumèta & Cola acuminata & Sterculaceae & 02 & 2,53 \\
\hline $\mathbf{0 9}$ & Gbandja & Cola nitida & Sterculaceae & 03 & 3,79 \\
\hline $\mathbf{1 0}$ & Ofu & Cola gigantea & Sterculaceae & 02 & 2,53 \\
\hline $\mathbf{1 1}$ & Ayoglin & Curculigopilosa & Hypoxidaceae & 04 & 5,06 \\
\hline $\mathbf{1 2}$ & Yéréoyimbo & Eugenia aromatica & Myrtaceae & 03 & 3,79 \\
\hline $\mathbf{1 3}$ & Kolla/orogbo & Garcinia kola & Clusiaceae & 12 & 15,18 \\
\hline $\mathbf{1 4}$ & Isassagbakoun & Monodora myristica & Annonaceae & 03 & 3,79 \\
\hline $\mathbf{1 5}$ & Abéré & Picralima nitida & Apocynaceae & 03 & 3,79 \\
\hline $\mathbf{1 6}$ & Yéréobè & Piper guineense & Piperaceae & 05 & 6,32 \\
\hline $\mathbf{1 7}$ & Aridan & Tetrapleura tetraptera & Mimosaceae & 02 & 2,53 \\
\hline $\mathbf{1 8}$ & Erun & Xylopia aethiopica & Annonaceae & 09 & 11,39 \\
\hline $\mathbf{1 9}$ & Ata ilè & Zingiber officinalis & Zingiberaceae & 04 & 5,06 \\
\hline $\mathbf{2 0}$ & Ata ilèkpukpa & Curcuma longa & Zingiberaceae & 04 & 5,06 \\
\hline $\mathbf{S 0 u}$ & & & &
\end{tabular}

Source : Enquêtes de terrain, mars 2018

Légende : Nr: Nombre; CPR : Contribution de chaque graine dans la constitution de recettes ou Fréquence d'implication de chacune des espèces de graines dans la constitution des recettes

De l'observation du tableau 1 , les espèces de graines comme Afframomum melegueta ( $\mathrm{CPR}=15,18)$, Garcinia kola (CPR $=15,18)$, Allium ascalonicum
$(C P R=11,39)$ et Xylopia aethiopica $(C P R=11,39)$ sont les plus impliquées dans la préparation de plusieurs recettes. Ces différentes espèces ont une 
importance capitale dans la constitution des recettes. Par contre, Antada gigas ( $C P R=1,26)$ contribue très faiblement dans la constitution des recettes.

Eléments d'association de quelques graines : Selon les enquêtés, la plupart des recettes en médecine traditionnelle exigent un ou plusieurs éléments d'association qui peuvent être des organes de plante, des liquides (eau, alcool) et huile ou encore des parties et/ou organes d'animaux. Le tableau 2 présente quelques maladies, remèdes suivant les graines sollicitées, leurs éléments d'association et le mode de préparation.

Tableau 2 : Maladies et remèdes suivant les graines sollicitées et leurs éléments d'association

\begin{tabular}{|c|c|c|c|c|}
\hline $\mathbf{N}^{\circ}$ & Noms scientifiques & Maladie & Eléments d'association & $\begin{array}{l}\text { Modes de } \\
\text { préparation }\end{array}$ \\
\hline 01 & Acacia siberiana & Règles douloureux & Alcool/ eau & Macération \\
\hline 02 & Afframomum melegueta & Hémorroïdes & $\begin{array}{l}\text { Escargots + feuilles du Ocimum } \\
\text { gratissimum+ huile palmiste }\end{array}$ & Cataplasme \\
\hline 03 & Allium ascalonicum & Paludisme & $\begin{array}{l}\text { Feuilles de Laurusnobilis + Jus du } \\
\text { Citrus limon }+ \text { alcool }\end{array}$ & Macération \\
\hline 04 & Allium cepa & Asthme & Eau + miel & Décoction \\
\hline 05 & Allium sativum & Hypertension & Miel + jus du Citrus limon + alcool & Macération \\
\hline 06 & Antada gigas & Gbagbla & Eau & Infusion \\
\hline 07 & $\begin{array}{l}\text { Chlorophyllum } \\
\text { blepharophyllum }\end{array}$ & Rougeole & Alcool & Macération \\
\hline 08 & Cola acuminata & $\begin{array}{l}\text { Protection } \\
\text { grossesse }\end{array}$ & Ecosse du cola acuminata + eau & Décoction \\
\hline 09 & Cola gigantea & Stérilité feminine & $\begin{array}{l}01 \text { gousse de Afframmomum } \\
\text { melegueta }+01 \text { poussin d'un jour }\end{array}$ & Cataplasme \\
\hline 10 & Cola nitida & Diarrhée & Alcool & Macération \\
\hline 11 & Curculigopilosa & Ernie & Potasse + Eau & Infusion \\
\hline 12 & Eugenia aromatica & Faiblesse sexuelle & $\begin{array}{l}10 \text { graines de Garcinia kola + } \\
\text { capsicum annum + alcool }\end{array}$ & Maceration \\
\hline 13 & Garcinia kola & Ulcère & Miel + jus du Citrus limon & $\begin{array}{ll}\begin{array}{l}\text { Pilage et } \\
\text { macération }\end{array} & \text { et } \\
\end{array}$ \\
\hline 14 & Monodora myristica & Courbature & $\begin{array}{l}\text { Xylopia aethiopica + Zingiber } \\
\text { officinalis+ huile rouge + gasoil }\end{array}$ & Pilage \\
\hline 15 & Picralima nitida & Hygiene genital & Beurre de Karité & Cataplasme \\
\hline 16 & Piper guineense & Ejaculation précoce & $\begin{array}{l}\text { Garcinia kola + Monodora myristica } \\
+ \text { Xylopia aethiopica + jus du Citrus } \\
\text { limon }\end{array}$ & Macération \\
\hline 17 & Tetrapleura tetraptera & Paludisme & Eau & Infusion \\
\hline 18 & Xylopia aethiopica & Hémorroïdes & Eau + Khaya senegalensis + sucre & Macération \\
\hline 19 & Zingiber officinalis & Paludisme & $\begin{array}{l}\text { Cymbopogon citratus }+ \\
\text { Laurusnobilist alcool }+ \text { fruit du } \\
\text { Citrus limon }\end{array}$ & Macération \\
\hline 20 & Curcuma longa & Constipation & Alcool & Macération \\
\hline
\end{tabular}

Source : Enquêtes de terrain, mars 2018

\| ressort de l'observation du tableau 2 que, les éléments d'association des graines recensées sont généralement constitués d'organes de plante. C'est l'exemple de Zingiber officinalis qui est associée aux feuilles de Cymbopogon citratus, Laurus nobilis, et des fruits de Citrus limon pour guérir le paludisme.

Modes de préparation des graines pour le traitement des affections: II a été recensé au cours des enquêtes de terrain, six (06) différents modes de 
préparation des graines médicinales commercialisées dans le marché de Adjarra-kpétou. La figure 4 présente les proportions des diverses formes pharmaceutiques de l'utilisation des graines médicinales pour le traitement de différentes affections.

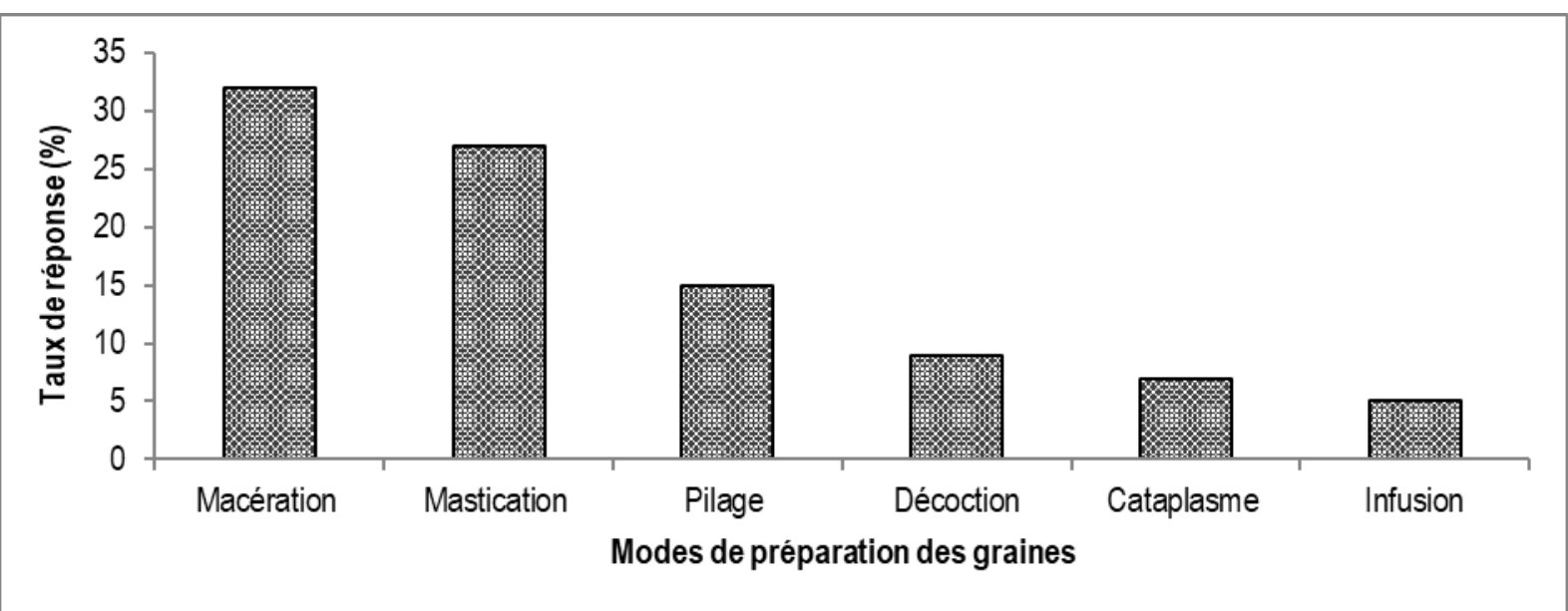

Figure 4 : Différents modes de préparation des graines

Source : Enquêtes de terrain, mars 2018

L'analyse de la figure 4 révèle que, la macération (aqueuse ou alcoolique) (32\%) et la mastication (27\%) sont les modes de préparation les plus utilisés dans la constitution des recettes à base de graines. Elles sont suivies du pilage (15\%), de la décoction $(9 \%)$, du cataplasme $(7 \%)$ et de l'infusion (5\%).De plus, selon les personnes sondées la meilleure façon de bénéficier des propriétés des graines serait de les macérer ou encore de les mastiquer directement.
Modes d'administration des préparations issues des graines: Tout comme les formes pharmaceutiques, plusieurs modes d'administration ont été aussi recensés lors des campagnes ethnobotaniques. II s'agit de la voie orale, l'usage externe qui regroupe bain et massage, et l'utilisation par les orifices. La figure 5 renseigne sur les proportions des modes d'administrations des recettes issues des graines recensées.

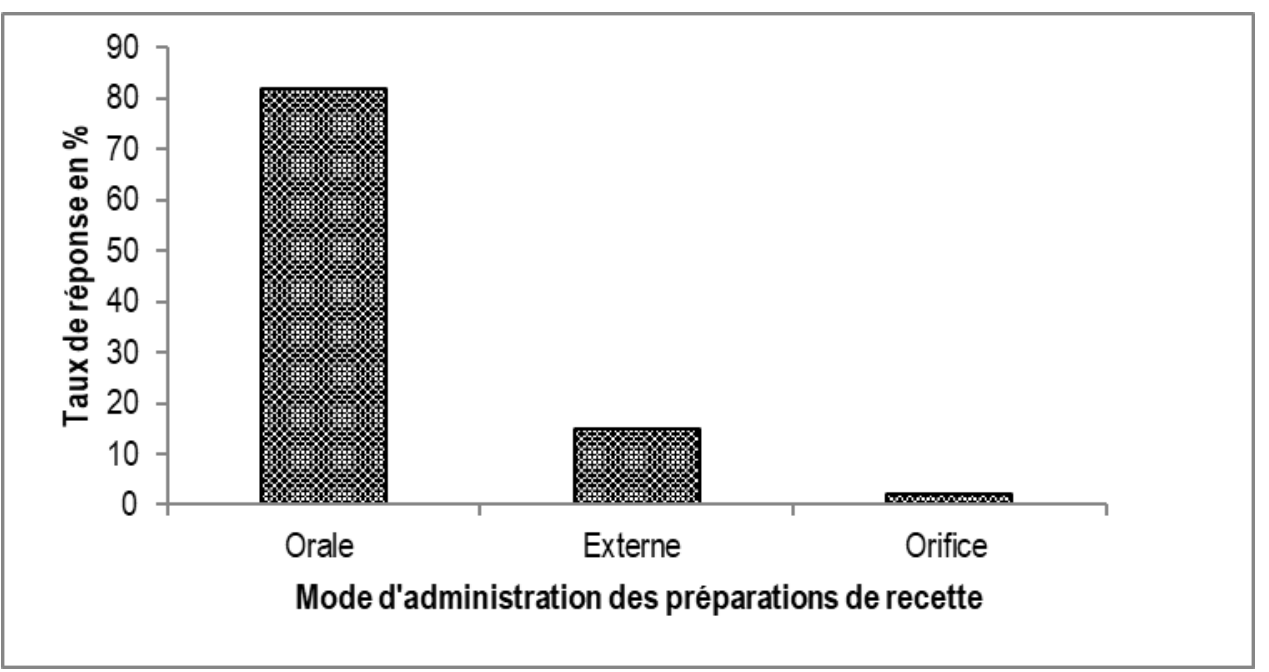

Figure 5 : Mode d'administration des préparations issues des graines

Source : Enquêtes de terrain, mars 2018

De l'analyse de la figure 5 , il ressort que la voie orale (82 \%) est le mode d'administration le plus cité.
Viennent, ensuite l'usage externe (bain et massage) $(16 \%)$ et l'utilisation par les orifices (2\%). Cette forte 
représentativité observée au niveau de la voie orale est fonction de la forte représentativité de la macération et de la mastication comme forme pharmaceutique.

Dosages des recettes dans le traitement des affections : Les personnes enquêtées ont fait mention la dose d'utilisation des préparations issues des graines. II s'agit des formes de dosage illustrées par la figure 6 .

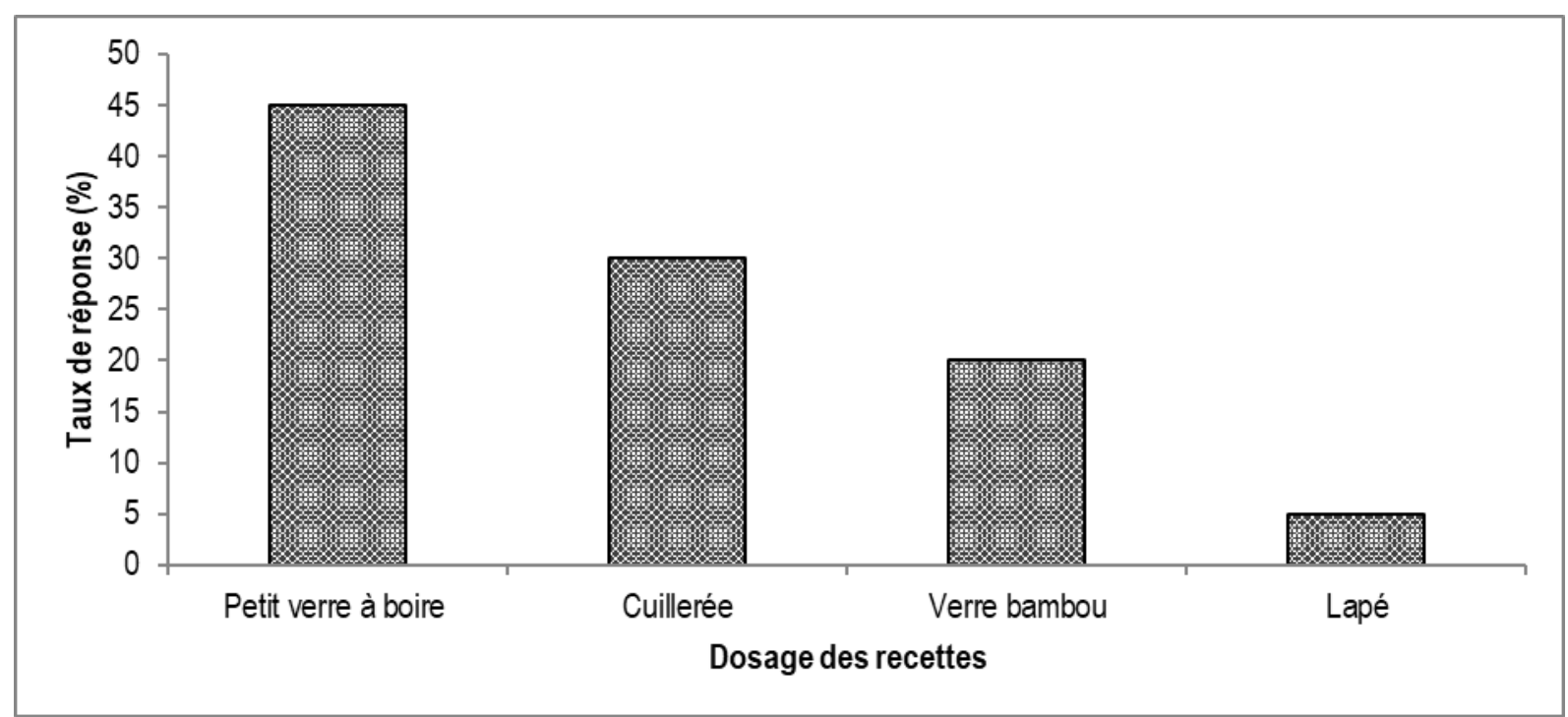

Figure6 : Dosage des recettes

Source : Enquêtes de terrain, mars 2018

D'après l'analyse de la figure 6 , il ressort que $45 \%$ des recettes issues de la préparation des graines médicinales sont consommées par le petit verre à boire. Par conséquent, le petit verre à boire est le dosage le plus indiqué pour la consommation des recettes issues de la préparation des graines médicinales dans la Commune de Adjarra.

Types d'affections traitées par les graines médicinales recensées: Un total de quarante-deux

(42) différentes affections ou symptômes ont été inventoriés auprès des différents acteurs (herboristes, acheteurs et guérisseurs traditionnels). Ces affections ont été par la suite classées en treize (13) différents types de maladies. La figure 7 présente ces types d'affections suivant les fréquences de référence à la médecine traditionnelle pour leur traitement. 


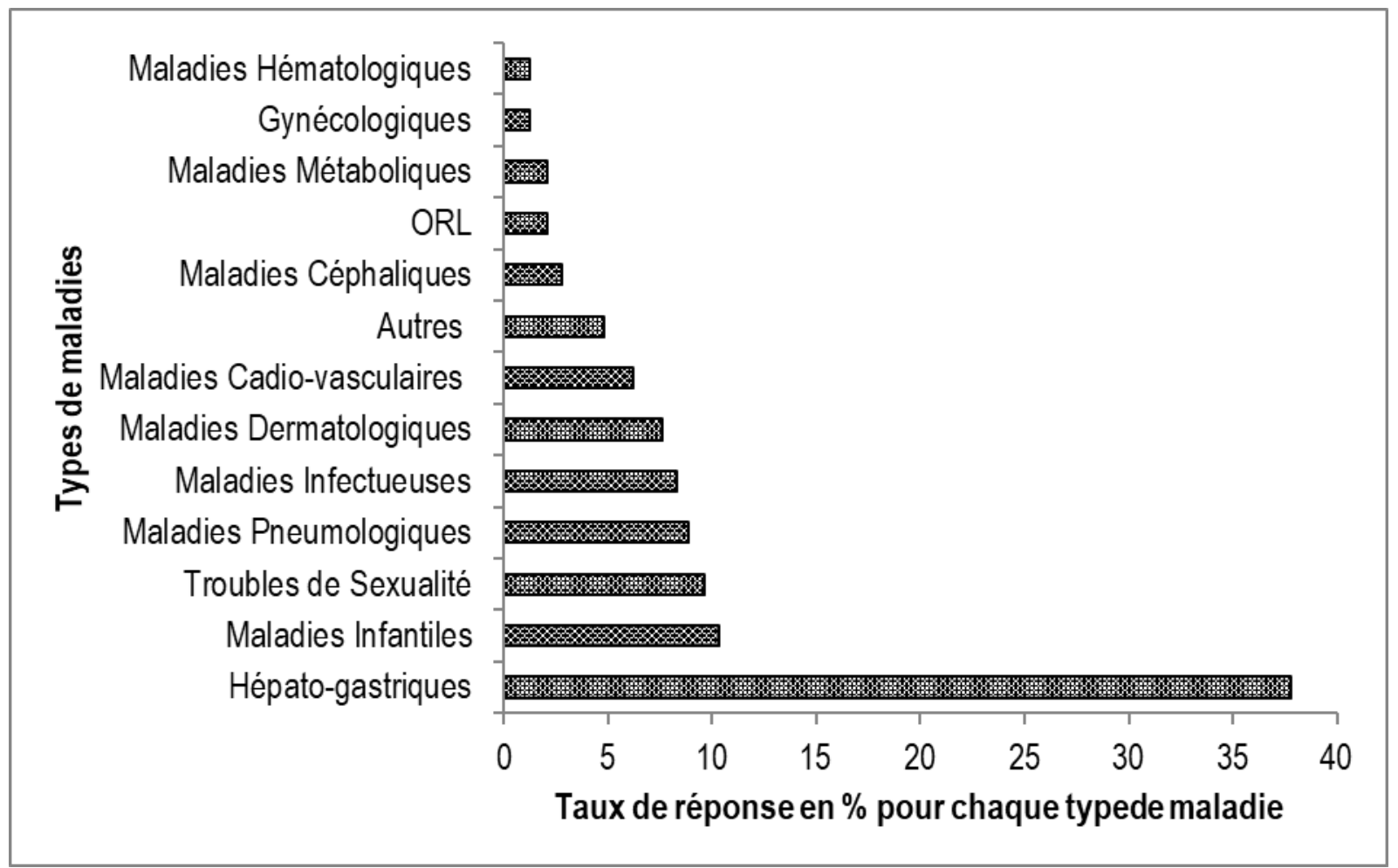

Figure 7 : Types de maladies et fréquence de référence à la médecine traditionnelle

Source : Enquêtes de terrain, mars 2018

D'après l'analyse de la figure 7 , il ressort que, les maladies de types hépato-gastriques (38 \%) et infantiles (10\%) sont les types pour lesquelles la population de la Commune de Adjarra fait plus fréquemment recourt à la médecine traditionnelle associée à l'utilisation des graines médicinales. Par contre, les maladies hématologiques et gynécologiques
(1\% chacune) sont faiblement citées par les acteurs enquêtés.

Groupes ethniques s'adonnant aux activités liées aux graines médicinales: II existe plusieurs groupes ethniques dans la Commune de Adjarra. Cependant, trois groupes s'adonnent à ce type d'activité. II s'agit, des Yoruba, des Nago et des Goun. La figure 8 montre la proportion d'apparition de ces groupes ethniques.

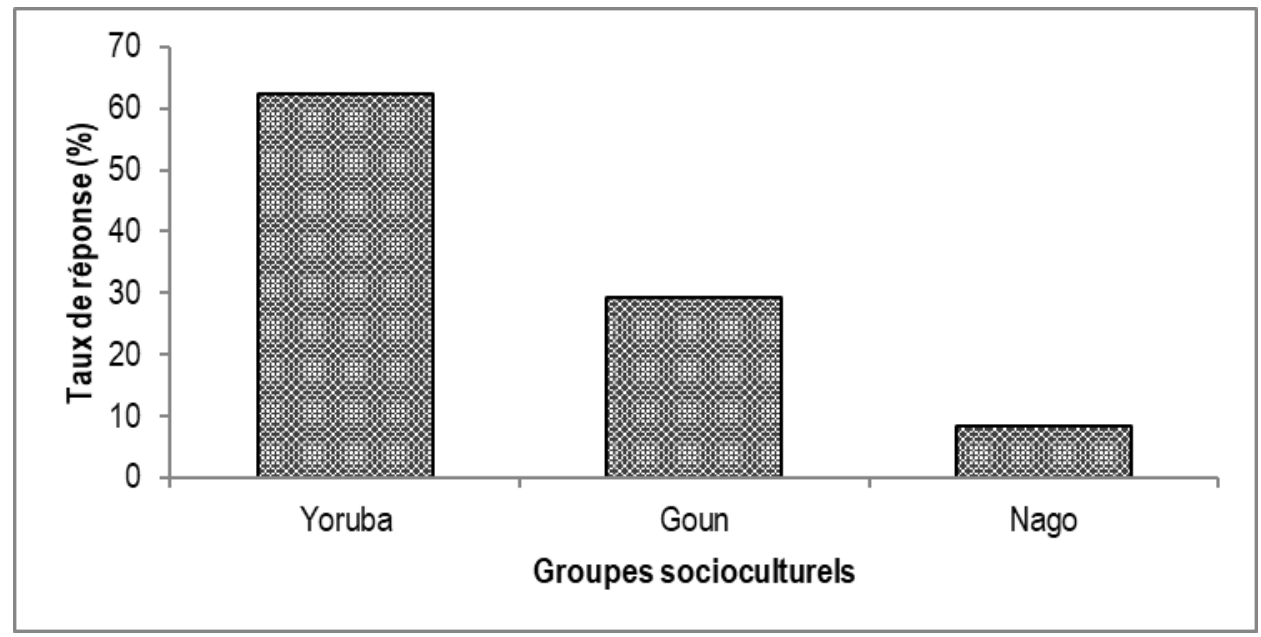

Figure 8 : Groupes ethnique

Source : Enquêtes de terrain, mars 2018 
L'analyse de la figure 8 révèle que les Yoruba $(62,5 \%)$ s'intéressent plus au commerce et à l'usage médicinal des graines médicinales, ensuite viennent les Goun $(29,16 \%)$ et enfin les Nago (8,33\%). Bien qu'étant

\section{DISCUSSION}

Le nombre d'espèces recensées dans cette étude (usage des graines à vocation médicinale commercialisées sur le marché de Adjarra-kpétou dans la Commune de Adjarra au sud-est du Bénin) confine avec les résultats de (Dibong et al., 2011.p2496) qui n'ont inventorié que trente (30) espèces sur 90 étales et ceci dans trois (03) marchés au Cameroun. De même, Gueye et al., (2012, p. 773) n'ont recensé que dix-huit (18) espèces, suite à une étude ethnobotanique portant sur les plantes utilisées dans le traitement de la constipation chez les Malinké du Sénégal. En revanche, nos résultats s'écartent largement de ceux obtenus par (Kouchadé et al., 2016, p. 4418) qui ont inventorié 272 espèces pour une étude ethnobotanique des plantes médicinales utilisées dans le traitement des maladies infantiles vendues sur les marchés du sud Bénin. Cette différence s'explique non seulement par le fait que, la présente étude n'a porté que sur les graines à vocation médicinale mais aussi du fait que ces derniers ont travaillé surtouts les marchés de la zone méridionale du Bénin. Les espèces de graines fréquemment citées pour le traitement des maladies appartiennent aux familles des Alliaceae, Mimosaceae, Sterculiaceae et des Zingiberaceae contrairement à plusieurs d'autres études ayant rapporté les Leguminoseae, Rubiaceae et Euphorbiaceae (Adomou et al., 2012, p. 745), Leguminoseae, Euphorbiaceae, Rubiaceae et Lamiaceae (Lougbégnon et al., 2015, p. 263) comme étant les familles contenant les espèces les plus utilisées dans le traitement des maladies en Afrique. La spécificité (choix des graines) de la présente recherche serait la raison fondamentale de cette grande dissemblance. Les espèces de graines recensées sont utilisées en tant que composantes principales pour traiter au total 42 affections regroupées en treize (13) types d'affections. En effet, les types de maladies les plus fréquemment citées sont: hépatogastriques (38\%), les maladies infantiles (10\%), et les troubles de sexualité $(10 \%)$. Cette étude surpasse donc l'information selon laquelle les graines ne sont qu'utilisées en tant qu'adjuvants ou ingrédients auxiliaires dans la préparation des recettes ou encore utilisées par plusieurs groupes ethniques, l'usage et surtout le commerce des graines médicinales est l'apanage des yoruba dans le secteur d'étude.

pour traiter les symptômes secondaires des maladies (Adomou et al., 2012, p. 748) et (Eddouks et al., 2007, p. 195). En ce qui concerne les formes pharmaceutiques (Macération $32 \%$ et mastication 20 $\%$ ), la présente étude se rapproche de celle menée par (Guèyè et al., 2012, p 775) ayant précisé que la macération est la forme pharmaceutique la plus fréquente $(57 \%)$ et que la décoction n'est utilisée que pour les feuilles. Elle s'oppose en contrario à plusieurs d'autres études montrant que la décoction est le mode de préparation le plus sollicité. II s'agit notamment de Kouchadé et al., (2016, p. 4418) rapportant la décoction à $93,48 \%$ à l'épilogue d'une étude ethnobotanique portant sur les plantes médicinales utilisées dans le traitement des maladies; de (Lougbégnon et al., 2015, p. 263) ayant rapporté à $75 \%$ la décoction; (Adomou et al., 2012, p. 745) à (86 \%) et (N'Guessan et al., 2009 , p. 42) à $(57,97 \%)$. Ces dissemblances sont dues au fait que la quasi-totalité de ces études ont porté sur les espèces en générale. Par ailleurs, il s'avère nécessaire de signaler que, l'usage des graines dans le traitement des maladies est l'un des moyens de sauvegarde de la biodiversité contrairement à l'emploie des organes tels les racines, les écorces portant préjudice à la suivie de l'espèce. Par exemple, Cassia siamea meurt suite à une récolte excessive de ses racines. Aussi, précisons que la meilleure façon de bénéficier des propriétés des graines serait de les macérer ou encore les masquer directement. La majorité des recettes médicamenteuses recensées durant cette étude est prise par voie orale ( $82 \%)$. Ce résultat, est supérieur à celui de (N'Guessan et al., 2009, p.42) dont l'étude pharmacologique sur les plantes antipaludiques utilisées en médecine traditionnelle chez les Abbey et Krobou d'Agboville (Côte d'lvoire) a montré que ce mode d'administration revient à 46,98\%.Ce résultat obtenu, est fonction du taux élevé des formes pharmaceutiques citées plus haut. Autrement dit, les médicaments issus de la macération sont généralement administrés par voie orale. A cela, s'ajoute la mastication qui consiste à consommer directement la graine. 


\section{CONCLUSION}

Cette étude a permis de constituer une base de données des connaissances traditionnelles des populations (maladies traitées, mode de préparation, mode d'administration et dosage) sur les principales graines à vocation médicinales commercialisées dans le marché de Adjarra-kpétou. Ainsi, quelques espèces de graines apparaissent comme plus importantes car sont plus impliquées dans la constitution de plusieurs recettes. Dans leur ordre d'importance il s'agit de:

\section{RÉFÉRENCE BIBLIOGRAPHIQUE}

Adjanohoun Joshua Edouard et Ake-Assi Laurent, 1979, Contribution au recensement des plantes médicinales de la Côte d'Ivoire. Centre National de Floristique : Abidjan ; 359 p.

Adomou Aristide Cossi, Yedomonhan Hounnankpon, Legba S. I, Oumoro Aliou Aboudou Madjidou, et Akoegninou Akpovi, 2012, Étude Ethnobotanique des plantes médicinales vendues dans le marché d'Abomey-Calavi au Bénin. Int. J. Biol. Chem. Sci. pp. 745-772.

Akoegninou Akpovi, Van Der Burg W. J et Van Der Maesen Laurentius Josephus Gerardus, Adjikedje V, Essou Jean-Pierre, Sinsin Brice Et Yedomonhan Hounnankpon, 2006, Flore Analytique du Bénin, pp. 1034

ASECNA, 2015. Station météorologique de Adjarra, Agence pour la Sécurité de la Navigation Aérienne en Afrique et à Madagascar.

Dassou Hospice Gbèwonmèdéa, Ogni Clément Abiola, Yedomonhan Hounnankpon, Adomou Aristide Cossi, Jiofack Taofick Réné Bernadin, Tossou Monique, Dougnon Tossou Jacques et Akoegninou Akpovi, 2014, Diversité, usages vétérinaires et vulnérabilité des plantes médicinales au Nord-Bénin Int. J. Biol. Chem. Sci. 8(1): 189-210

Dibong Siegfried. Didier, Mpondo Emmanuel, Ngoye Alfred, Kwin marie-France, Betti Jean Lagarde, 2011, Ethnobotanique et phytomédecine des plantes médicinales de Douala, Cameroun. Journal of Applied Biosciences, 37: 2496 2507.

Dougnon Tamègnon Victorien, Attakpa Eugène, Bankole Honoré, Hounmanou Yaovimahou Gildas, Dehou René, Agbankpe Jérrold, De Souza Muriel, K. Fabiyi, Gbaguidi Fernand, Baba-Moussa Lamine, 2016, Etude ethnobotanique des plantes médicinales utilisées contre une maladie cutanée
Afframomum melegueta, Garcinia kola, Xylopia aethiopica, Allium sativum et Zingiber officinalis. Ces espèces devront donc fait partir des espèces de graines à sauvegarder et/ou analysées au laboratoire pour en extraire les principes actifs. De plus, l'utilisation thérapeutique des espèces de graines est aujourd'hui aperçue comme un moyen inébranlable pour la sauvegarde de la biodiversité.

contagieuse: La gale humaine au Sud-Bénin, pp. 16-22

Eddouks Mohamed, Ouahidi Mohammed, Farid Omra, Moufid Abderrahmane, Khalidi Aelzhe, Lemhadri Ahmed, 2007, L'utilisation des plantes médicinales dans le traitement du diabète au Maroc. Phytothérapie, p. 194-203

Fatiha El Azzouzi, Lahcen Zidane, 2015, La flore médicinale traditionnelle de la région de BéniMellal (Maroc) Journal of Applied Biosciences 91:8493 - 8502

Fokunang Charles, Guedje Nicole Marie, Kemeuze Victor, Fongnzossie Evariste, Nkongmeneck Bernad-Aloys, Mapongmetsem Pierre Marie, Tsabang No le, 2010, Ethnobotanical uses of medicinal plants of two ethno ecological regions of Cameroon, $44 \mathrm{p}$.

Gnagne Arthur Stéphane, Camara Djeneb, Fofie N'guessan Bra Yvette, Bene Kouadio Et Zirihi Guédé Noël, 2017, Étude ethnobotanique des plantes médicinales utilisées dans le traitement du diabète dans le Département de Zouénoula (Côte d'Ivoire) Journal of Applied Biosciences 113: 11257-11266

Gueye Mathieu, Cisse Ababacar, Diatta Cheikh Daouda, Diop Seydina, Koma Souleye, 2012, Etude ethnobotanique des plantes utilisées contre la constipation chez les Malinké de la communauté rurale de Tomboronkoto, Kédougou (Sénégal) Int. J. Biol. Chem. Sci. 6(2): 773-781,

Kone Mamidou Witabouna, Atindehou Kamanzi, Tere Henri, Traoré Dossahoua, 2002, Quelques plantes médicinales utilisées en pédiatrie traditionnelle dans la région de Férékessé dougou, (Côte d'Ivoire). Actes du colloque international, Centre Suisse du 26-27 Août 2001. BIOTERRE, Revue Internationale des Sciences de la Vie et de la Terre, $n^{\circ}$ spécial, 
2002. Editions Universitaires de Côte d'Ivoire, $30: 30-36$

Kouchade Aribikè Sabine, Adomou Aristide Cossi, Tossou G. Monique, Yedomonhan Hounnankpon, Et Dassou Hospice, Akoegninou Akpovi, 2016, Étude ethnobotanique des plantes médicinales utilisées dans le traitement des maladies infantiles et vendues sur les marchés au sud du Bénin, pp. 4418-4438.

Lougbégnon Olou Toussaint, Gbesso G H François, Codjia Jean-Claudethomas, 2015, Diversité et forme d'utilisation des plantes médicinales vendues sur les marchés de la Commune de Ouidah au Sud du Bénin. Revue de géographie du laboratoire Lied - ISSN 0851 $2515-N^{\circ} 13$, décembre 2015.

Mpondo Emmanuel, Yinyang Jacques, Dibong Siegfried Didier, 2015, Valorisation des plantes médicinales à coumarines des marchés de Douala Est (Cameroun), p. 78047823

N'guessan Koffi, Honora Tra Bi Fezan, Kone Mamidou Witabouna, 2009, Étude ethnopharmacologique des plantes antipaludiques utilisées en médecine traditionnelle chez les Abbey et Kroboud'Agboville (Côte d'Ivoire) 42-50pp

Ngene Jean-Pierre, Ngoule Charles Christian, Pouka Kidik Catherine-Marie, Mvogo Ottou Patrice Brice, Ndjib Rosette Christelle, Dibong Siegfried Didier, Mpondo Mpondo Emmanuel, 2015, Importance dans la pharmacopée traditionnelle des plantes à flavonoïdes vendues dans les marchés de Douala est (Cameroun), p. 8194-8210

Okafor Jonathan, Ham Rebecca, 1999, Identification, utilization, and conservation of medicinal plants in southeastern Nigeria. Issues in AfricaBiodiversity3: 1-7.

Tardio Javier, Pardo-De-Santayana Manuel, 2008, Cultural Importance Indice: A Comparative Analysis Based on the Useful Wild Plants of Southern Cantabria (Northern Spain). Economic Botany 62, 24 -39.

Weze L Alexander, 2001, Plantes médicinales et leur utilisation traditionnelle chez les paysans au Niger. Etudes sur la flore et la végétation du Burkina Faso et des pays avoisinants. Vol. 6. Editions Verlag Nature et Wissenschaft, Solingen Francfort et Ouagadougou ; 9-18.
Zerbo Patrice, Millogo-Rasolodimby Jeanne, NacoulmaOuedrago Odile, Van Damme Patrick, 2007, Contribution à la connaissance des plantes médicinales utilisées dans les soins infantiles en pays San, au Burkina Faso. Int. J. Biol. Chem. Sci. 1(3) :262-274. 